\title{
Machine Learning based Energy Management at Internet of Things Network Nodes
}

\author{
Dr. Joy Iong Zong Chen, \\ Professor, Department of Electrical Engineering, \\ Da-Yeh University, Taiwan. \\ Email id: jchen@mail.dyu.edu.tw \\ Kong-Long Lai, \\ Department of Electrical Engineering, \\ Da-Yeh University, Taiwan.
}

\begin{abstract}
The Internet of Things networks comprising wireless sensors and controllers or IoT gateways offers extremely high functionalities. However, not much attention is paid towards energy optimization of these nodes and enabling lossless networks. The wireless sensor networks and its applications has industrialized and scaled up gradually with the development of artificial intelligence and popularization of machine learning. The uneven network node energy consumption and local optimum is reached by the algorithm protocol due to the high energy consumption issues relating to the routing strategy. The smart ant colony optimization algorithm is used for obtaining an energy balanced routing at required regions. A neighbor selection strategy is proposed by combining the wireless sensor network nodes and the energy factors based on the smart ant colony optimization algorithm. The termination conditions for the algorithm as well as adaptive perturbation strategy are established for improving the convergence speed as well as ant searchability. This enables obtaining the find the global optimal solution. The performance, network life cycle, energy distribution, node equilibrium, network delay and network energy consumption are improved using the proposed routing planning methodology. There has been around $10 \%$ energy saving compared to the existing state-of-the-art algorithms.
\end{abstract}

Keywords: Internet of Things; Machine Learning; Ant Colony Optimization; Energy Management; WSN;

\section{Introduction}

With the rapid development in internet access, information processing and mature sensor network technologies, the network information, communication and innovation technology industries have launched the new industrial model based on Internet of Things (IoT) [1]. Irrespective of the region of operation, interconnection of information of all things or devices is achieved at all times as the main goal of IoT. The mainstream technologies like wireless communication, computer, embedded and sensor technology are integrated using the wireless sensor network (WSN). Wide collection range, no wiring and low cost are the main characteristics of this system [2]. Military investigation, biomedical, smart home, environmental monitoring, early warning for natural disaster and various such fields and unknown environments exploit the perception capabilities and the cognition abilities of IoT. Software technologies, network security, information processing, hardware technologies, smart embedded systems, WSN, Radio Frequency Identification Devices (RFID) and several such technologies are analyzed by various researchers in relevance to the implementation of IoT. The IoT sensing layer comprises of WSN and RFID technologies. This layer comprises of a network of sensor nodes that performs data collection and transmission [3].

Specific routing rules must be followed by data in the network. The mode of data transmission, type of heterogeneous node, dynamic network topology and such specific factors affect the formulation of IoT routing rules [4]. The industry scholars in scientific research are focused on developing better IoT routing algorithms. Designing a unified routing protocol that is appropriate for IoT based operations is of great research prospect. 
Journal of trends in Computer Science and Smart technology (TCSST) (2020)

Vol.02/ No. 03

Pages: $127-133$

https://www.irojournals.com/tcsst/

DOI: https://doi.org/10.36548/jtcsst.2020.3.001

Currently, as the standard system and IoT architecture are not unified, it is not possible to establish the routing strategy effectively in the IoT environment [5]. Specific applications are implemented with suitable IoT routing algorithms as proposed by various scholars performing research over the routing protocols of IoT. The efficient dynamic routing strategy in use works on the following principle- with the occurrence of a failed node in a system, a new parent node is found by the child node [6]. Path breakage is prevented by connecting the child node by broadcasting. Network energy is conserved by combining the low energy consuming nodes to the network as parent nodes based on the research recommendations. Large-scale properties cannot be implemented due to the simplicity of the network despite being able to extend the network life cycle. Reliability and stability cannot be assured due to the complex nature of IoT. Appropriate addressing strategies have been proposed for IoT by various researchers due to its large scale [7]. Routing prefixes are introduced for load balancing.

\section{Related Works}

An efficient mechanism for data transmission is provided for the upper IoT layers by designing a routing protocol at the IoT perception layer network that will be appropriate for the heterogeneous networks [8]. The network data transmission failure rate is reduced while the virtual pipelines execute multi-path transmission as a logical technique for multi-path pipeline. The multi-path data transmission issue is addressed by isolating the individual transmission path with the help of pipe [9]. A huge quantity of energy is consumed while entirely disjoint multipaths are built. The network energy consumption is balanced, path reliability is improved, and single path transmission tasks are shared using the multi-path routing algorithms [10-11]. Route maintenance and path switching can be improved further while multipath management is mostly neglected by the existing algorithms.

The adaptive property of ant colony optimization algorithms have been used for developing protocols in the distributed multi-path routing algorithm [12]. The node hoops and remaining energy are the key factors in this algorithm. The network life cycle is extended by choosing a path with weights similar to that of the optimal path, having largest energy and smallest hop count using a balanced multi-routing algorithm. The WSN node energy consumption issue is addressed in this paper using a novel routing algorithm that uses extended smart ant colony optimization algorithm. Here, the next hop of the node is selected within the network area by dividing the best area on the basis of delay and energy [13]. The ant's optimal position is proposed for ensuring the global optimal solution. The algorithm's convergence speed can be accelerated using the termination condition strategy of the proposed algorithm. The simulation results prove the efficiency of this algorithm [14]. 
Journal of trends in Computer Science and Smart technology (TCSST) (2020)

Vol.02/ No. 03

Pages: $127-133$

https://www.irojournals.com/tcsst/

DOI: https://doi.org/10.36548/jtcsst.2020.3.001

\section{Proposed Work}

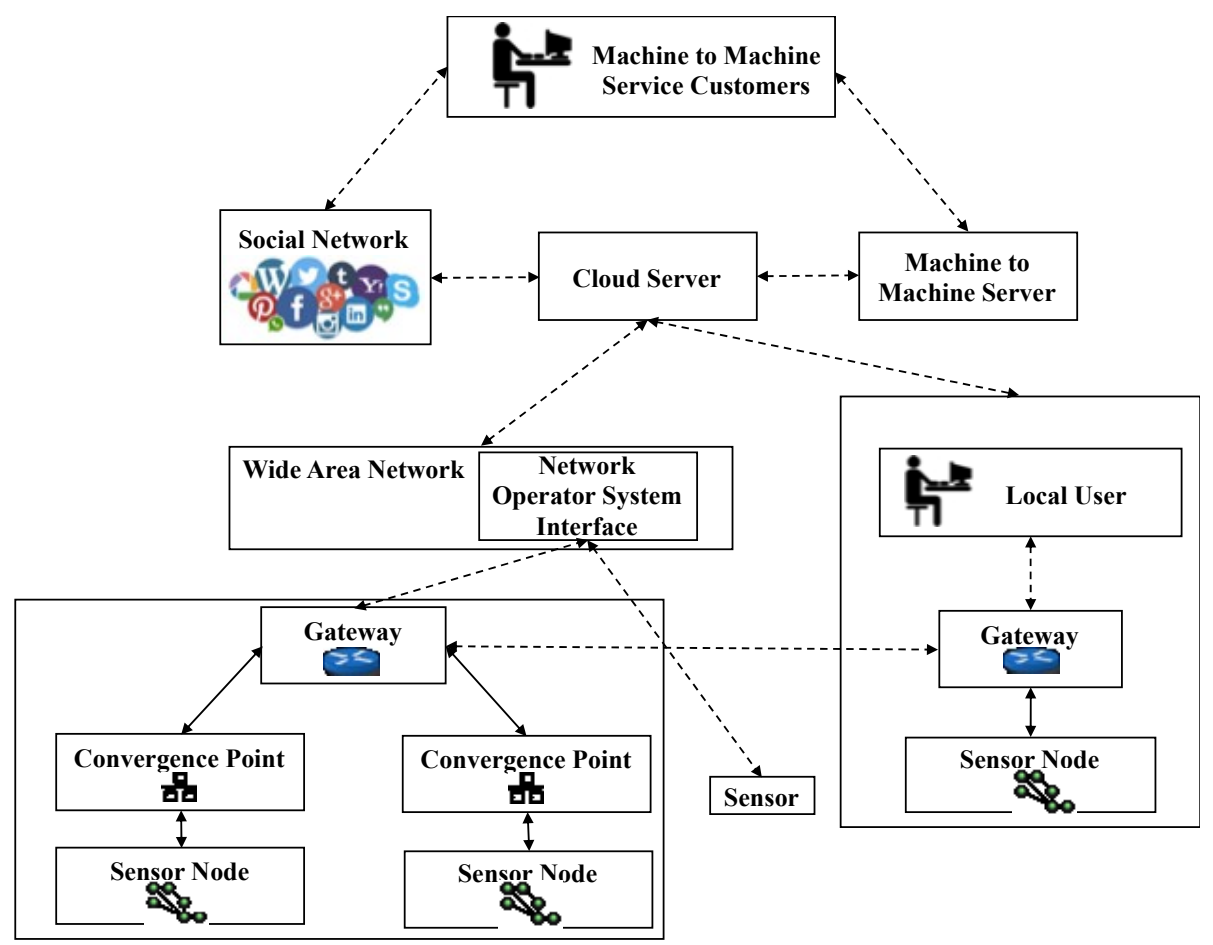

Figure 1: Machine to Machine Architecture of the IoT network nodes

The network capabilities and communication of equipment and machine is enhanced by a technology collectively termed as Machine to Machine (M2M) network. Mobile internet communication, human-machine interactive communication, machine control communication and other such communication technologies between the machines can be combined using the M2M network. Information from the application processes, equipment and machines are shared with the operators and the background information systems. Without the need for direct human intervention, the establishment of communication among machines is the fundamental principle of M2M network. The decision making and communication problem is attempted to be completed automatically in M2M network business. Wireless interfaces are used for enabling communication of smart devices with each other. The master computers are used for establishing a communication connection.

The application domain, network domain and M2M domain are the major constituents of the M2M network architecture. The gateways and nodes of the M2M domain is as represented in Figure 1. The communication, protocol conversion, data aggregation and collection functions can be performed completely by the M2M domain. The transmission of information from the M2M to the application domain can be performed reliably through the channel provided by the network domain. The M2M protocol manages and maintains the complete transmission network based on the mobile and internet communication. The remote management and monitoring applications based on M2M stores the uploaded information and offers real time data in the M2M domain which acts as a back-end service platform for the application domain. Analysis, processing and control of the gathered information is performed based on the requirements of various applications and the decision-making process is executed. 
Journal of trends in Computer Science and Smart technology (TCSST) (2020)

Vol.02/ No. 03

Pages: $127-133$

https://www.irojournals.com/tcsst/

DOI: https://doi.org/10.36548/jtcsst.2020.3.001

Ant foraging process is the basic principle of the Ant Colony Optimization (ACO) algorithm. The biological pheromone is replaced by artificial pheromone and the natural ant by the artificial ant in the algorithm. The concentration of the artificial pheromone is inversely proportional to the path length in the basic ACO. The higher concentration path is chosen by more artificial ants in this algorithm. Shortest path is chosen when the algorithm reaches the specified number of iterations. The basic ants in the ACO are of two types namely pathfinding and fallback ants. They originate from the source node and fall bac to the source from the destination node respectively.

\section{Results and Discussion}

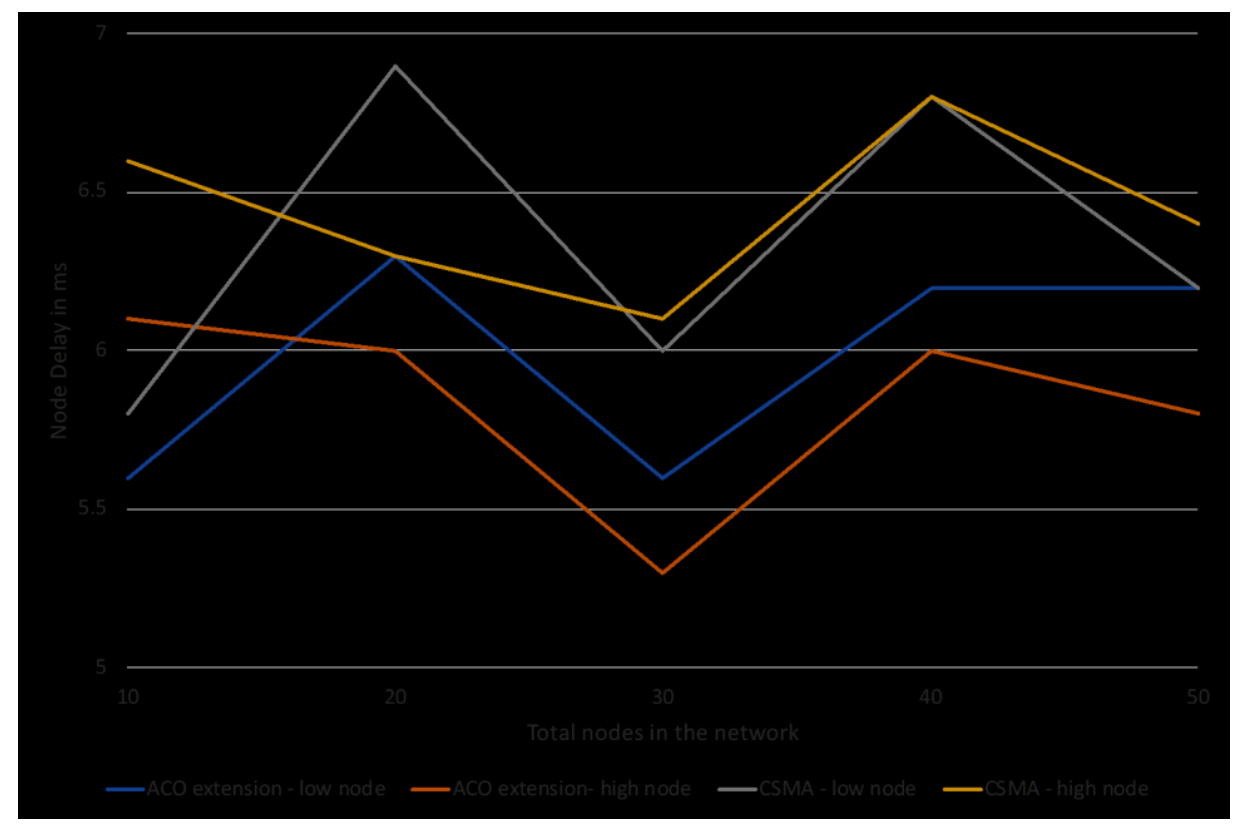

Figure 2: Comparison of the node delays sent by the extended ant colony optimization algorithm

The network energy consumption is reduced by transmitting over the maximum distance within the nodes communication range in an ideal setup. However, the perceived network and its real time performance is affected due to a large delay in data forwarding caused by the large number of participating nodes. The network delay can be reduced efficiently with data transmission. Energy holes are generated when large amount of energy is consumed by a single node. The routing design of the energy constrained WSN and its requirements are not met in this case. The energy delay measures are compared with the range of interval of SR and LR algorithm above the two ideal states. 
Journal of trends in Computer Science and Smart technology (TCSST) (2020)

Vol.02/ No. 03

Pages: $127-133$

https://www.irojournals.com/tcsst/

DOI: https://doi.org/10.36548/jtcsst.2020.3.001

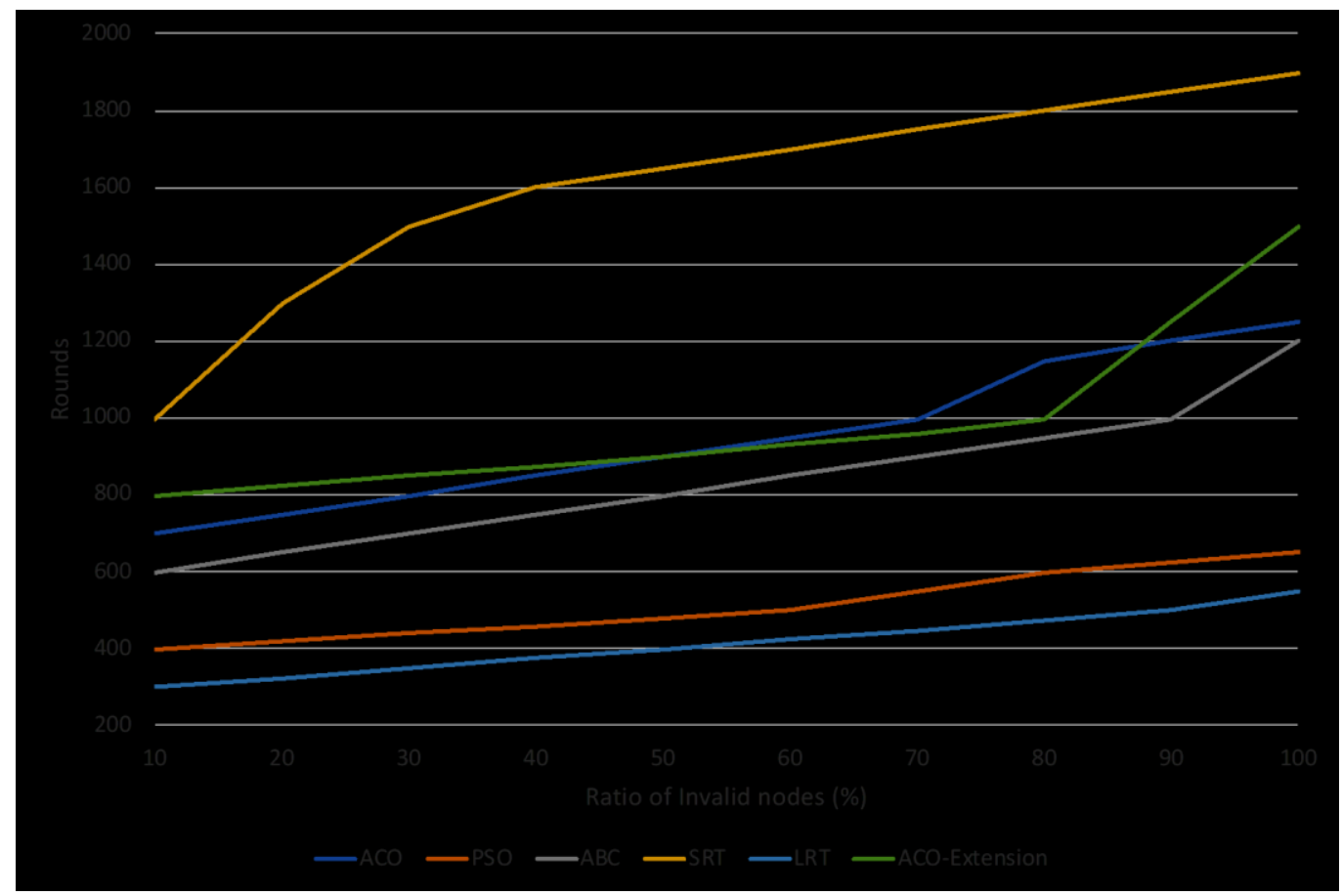

Figure 3: Comparison of the relationship between the proportion of failed nodes and the number of rounds of the algorithm

Figure 2 represents the comparison of the node delays sent by the extended ant colony optimization algorithm. Within the specified communication range, data transmission to the nearest node is performed by the SR node. There is an optimal number of communication rounds with the reduction in the single node energy consumption when the participating nodes are in large number. Transmission is performed by the LR using nodes to the farthest nodes in the range of communication. Figure 3 represents the comparison of the relationship between the proportion of failed nodes and the number of rounds of the algorithm. The change in packet size of transmission data and energy delay are compared for the six algorithm protocols. Delay can be characterized by the energy delay measure as the delay and energy magnitude vary greatly. When delay is taken into consideration, the number of hops and delay are related. Hence, LR algorithm is preferred due to the availability of large number of nodes. Figure 4 provides the comparison of algorithm data packet size and delay.

The CSMA/CA protocol and the extended smart ant colony algorithm are used by the star M2M network. The transmission delay of the high energy consuming network nodes are reduced using the extended smart ACO algorithm in M2M network. In certain cases, there is a staggering between the delay in transmission of the high and low energy consuming nodes. The lower energy nodes have larger transmission delay than that of higher energy nodes. A stable transmission delay is adopted by the M2M network with the increase in the number of network nodes. 
Journal of trends in Computer Science and Smart technology (TCSST) (2020)

Vol.02/ No. 03

Pages: $127-133$

https://www.irojournals.com/tcsst/

DOI: https://doi.org/10.36548/jtcsst.2020.3.001

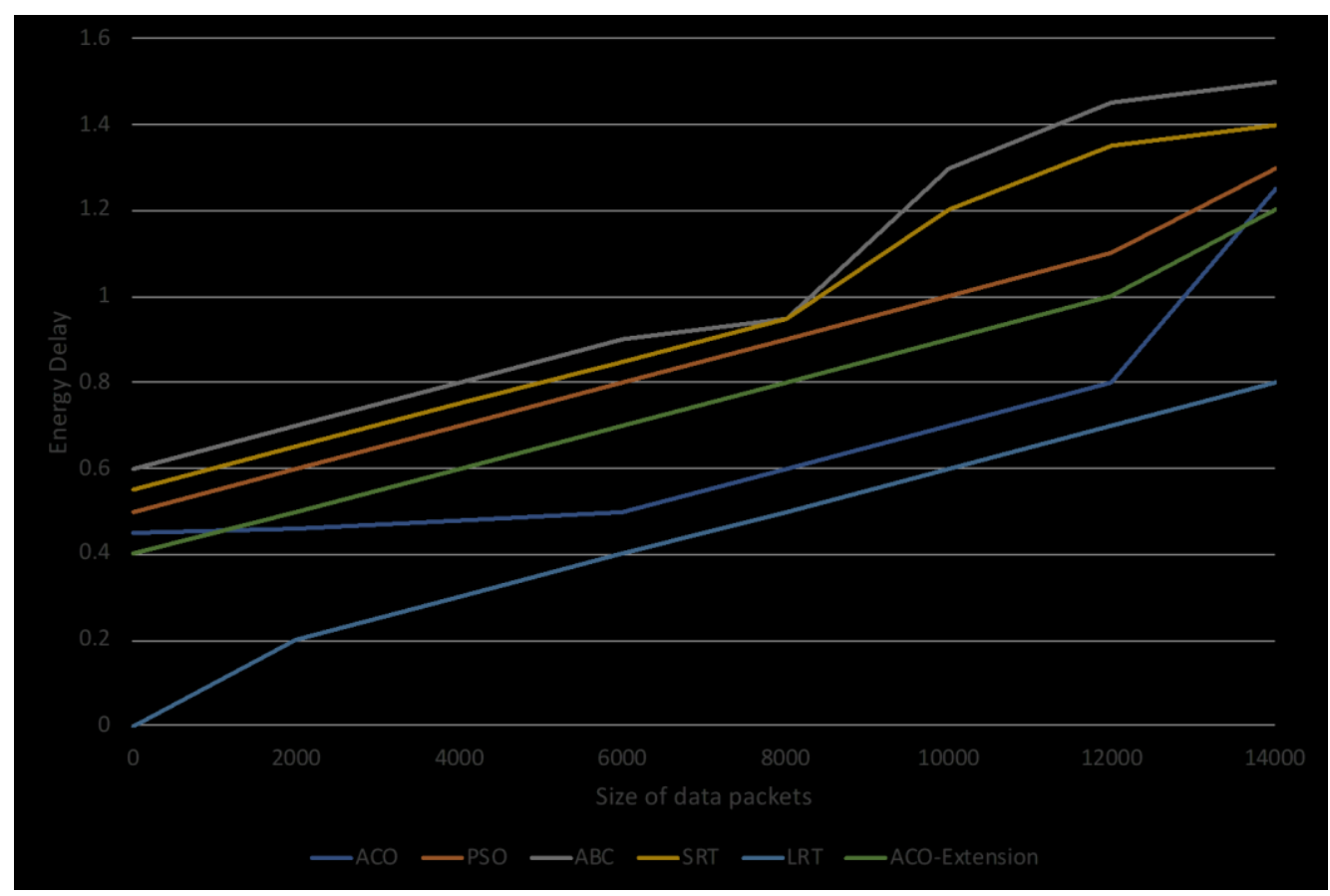

Figure 4: Comparison of algorithm data packet size and delay

\section{Conclusion}

There is tremendous growth in the field of IoT with the development of smart devices and technology. Research in the wireless sensor networks, artificial intelligence, and IoT has gained prominent importance in the recent days. Energy optimization in the network nodes is essential as it is possible that local optimum is reached by easy routing algorithms that leads to uneven consumption of energy at the nodes of the network when the routing protocols are used. This can be overcome by using smart optimization algorithm like the ACO and performing dynamic optimization. The energy consumption of the network is optimized and a global optimal solution is found by dividing the areas based on the delay and energy using smart ant colony optimization and planning protocol for routing. Machine learning is used for studying the energy patterns and in further reducing the energy consumption in these networks. The jump probability is combined on the basis of the node location and the node transmission area which is divided to explore the potential nodes considering the delay and energy of the divided area. The global optimal solution is derived using the characteristics of termination conditions of the algorithm, adaptive perturbation strategy, neighbor selection strategy and wireless sensor network in combination with the smart ant colony optimization algorithm. Along with the ACO, PSO, ABC, SRT, LRT and ACO extension algorithms are compared for network performance measurements and the energy delay metric analysis. Balanced consumption of energy is obtained on implementation of this algorithm along with network life cycle extension in an efficient manner. The delay and energy consumption of the sensor network are considered and the network node energy consumption is balanced. The delay and energy issues are solved by the multiple mobile agents even in large scale networks. Future work is directed towards analysis of coordination between the multiple mobile agents. 
Journal of trends in Computer Science and Smart technology (TCSST) (2020)

Vol.02/ No. 03

Pages: $127-133$

https://www.irojournals.com/tcsst/

DOI: https://doi.org/10.36548/jtcsst.2020.3.001

\section{References}

[1] Liu, X., Zhao, S., Liu, A., Xiong, N., \& Vasilakos, A. V. (2019). Knowledge-aware proactive nodes selection approach for energy management in Internet of Things. Future generation computer systems, 92, 1142-1156.

[2] Spanias, A. S. (2017, August). Solar energy management as an Internet of Things (IoT) application. In 2017 8th International Conference on Information, Intelligence, Systems \& Applications (IISA) (pp. 1-4). IEEE.

[3] Mydhili, S. K., Periyanayagi, S., Baskar, S., Shakeel, P. M., \& Hariharan, P. R. (2019). Machine learning based multi scale parallel K-means++ clustering for cloud assisted internet of things. Peer-to-Peer Networking and Applications, 1-13.

[4] Kumar, S., Solanki, V. K., Choudhary, S. K., Selamat, A., \& González Crespo, R. (2020). Comparative Study on Ant Colony Optimization (ACO) and K-Means Clustering Approaches for Jobs Scheduling and Energy Optimization Model in Internet of Things (IoT). International Journal of Interactive Multimedia \& Artificial Intelligence, 6(1).

[5] Khattab, A., \& Youssry, N. (2020). Machine Learning for IoT Systems. In Internet of Things (IoT) (pp. $105-$ 127). Springer, Cham.

[6] Bui, K. H. N., \& Jung, J. J. (2019). ACO-based dynamic decision making for connected vehicles in IoT system. IEEE Transactions on Industrial Informatics, 15(10), 5648-5655.

[7] Hossain, E., Khan, I., Un-Noor, F., Sikander, S. S., \& Sunny, M. S. H. (2019). Application of big data and machine learning in smart grid, and associated security concerns: A review. IEEE Access, 7, 13960-13988.

[8] Ashaj, S. J., \& Erçelebi, E. (2020). Energy Saving Data Aggregation Algorithms in Building Automation for Health and Security Monitoring and Privacy in Medical Internet of Things. Journal of Medical Imaging and Health Informatics, 10(1), 204-210.

[9] Bogale, T. E., Wang, X., \& Le, L. B. (2018). Machine intelligence techniques for next-generation contextaware wireless networks. arXiv preprint arXiv:1801.04223.

[10] Manshahia, M. S. (2018). Swarm intelligence-based energy-efficient data delivery in WSAN to virtualise IoT in smart cities. IET Wireless Sensor Systems, 8(6), 256-259.

[11] Darwish, A., Hassanien, A. E., Elhoseny, M., Sangaiah, A. K., \& Muhammad, K. (2019). The impact of the hybrid platform of internet of things and cloud computing on healthcare systems: opportunities, challenges, and open problems. Journal of Ambient Intelligence and Humanized Computing, 10(10), 4151-4166.

[12] Suma, V. (2019). Towards sustainable industrialization using big data and internet of things. Journal of ISMAC, 1(01), 24-37.

[13] Karthiban, M. K., \& Raj, J. S. (2019). Big data analytics for developing secure internet of everything. Journal of ISMAC, 1(02), 129-136.

[14] Raj, J. S. (2019). QoS optimization of energy efficient routing in IoT wireless sensor networks. Journal of ISMAC, 1(01), 12-23. 